\title{
Assessment of Coronary Plaque Vulnerability in Acute Coronary Syndromes using Optical Coherence Tomography and Intravascular Ultrasound. A Systematic Review
}

\author{
Theodora Benedek, András Mester, Annabell Benedek, Nora Rat, Diana Opincariu, Monica Chițu \\ Department of Cardiology, University of Medicine and Pharmacy, Tîrgu Mureş, Romania
}

\begin{abstract}
The aim of this systematic review was to analyze studies characterizing vulnerable coronary plaques using optical coherence tomography (OCT) and intravascular ultrasound (IVUS), in order to identify the most efficient invasive technique permitting plaque characterization in patients with acute myocardial infarction. Method: A total number of 432 studies were identified, 420 through database searching and 12 through manual searching. Eight duplicate studies were removed, leaving a total number of 424 studies to be screened. Twenty-six studies only available in abstract-only form were excluded, resulting in 398 studies checked for eligibility. Eleven studies fulfilled the eligibility criteria and were included in this systematic analysis. Plaque vulnerability was investigated in plaques with thin cap fibroatheroma (TCFA) versus those with thick cap fibroatheroma, in ruptured coronary plaques versus non-ruptured coronary plaques, in culprit versus non-culprit lesions and in lipid-rich versus non-lipid-rich plaques. Results: A total of 1,568 coronary plaques in 1,225 patients with acute coronary syndromes (ACS) who underwent both IVUS and OCT for analysis of plaque features were included in the final analysis. The review identified the following IVUS-derived features as significantly correlated with plaque vulnerability: plaque burden $(\mathrm{p}<0.001)$, remodeling index $(\mathrm{p}<0.001)$, external elastic membrane cross-sectional area $(\mathrm{p}<0.001)$, and the amount of necrotic core $(\mathrm{p}<0.001)$, while OCT-derived features characterizing unstable plaque were TCFA ( $\mathrm{p}<0.001)$, lipid arch ( $\mathrm{p}$ $<0.001$ ), accumulation of macrophages ( $p=0.03$ ), and presence of intracoronary thrombus ( $p$ $<0.001)$. Conclusion: Both IVUS and OCT are invasive imaging techniques able to provide relevant information on the vulnerability of coronary atheromatous plaques, identifying, as they do, various plaque features significantly associated with unstable plaques. Information provided by the two techniques is complementary, and both methods can serve as a useful clinical diagnostic tool, especially in cases of ACS patients undergoing a revascularization procedure.
\end{abstract}

Keywords: vulnerable plaque, acute coronary syndrome, invasive coronary imaging, culprit plaque

\section{ARTICLE HISTORY}

Received: 10 October, 2016

Accepted: 20 November, 2016

\section{CORRESPONDENCE}

Annabell Benedek

Str. Gheorghe Marinescu nr. 38

540139 Tîrgu Mureș, Romania

Tel: +40 265215551

E-mail: annabell.benedek@yahoo.com

Theodora Benedek: Str. Gheorghe Marinescu nr. 38, 540139 Tîrgu Mureș, Romania. Tel: +40 265215 551. E-mail: theodora.benedek@gmail.com András Mester: Str. Gheorghe Marinescu nr. 38, 540139 Tîrgu Mureș, Romania. Tel: +40 265 215 551. E-mail: andras.mester@yahoo.com Nora Rat: Str. Gheorghe Marinescu nr. 38, 540139 Tîrgu Mureș, Romania. Tel: +40 265215 551. E-mail: ratnora@gmail.com Diana Opincariu: Str. Gheorghe Marinescu nr. 38, 540139 Tîrgu Mureș, Romania. Tel: +40 265 215 551. E-mail: diana.opincariu@yahoo.ro Monica Chițu: Str. Gheorghe Marinescu nr. 38, 540139 Tîrgu Mureș, Romania. Tel: +40 265215 551. E-mail: iuliachitu@yahoo.com 


\section{INTRODUCTION}

Acute coronary syndromes (ACS) are a major healthcare problem, as they are currently responsible for the majority of cardiac deaths worldwide. Despite significant efforts for the implementation of preventive measures to reduce the burden of cardiovascular diseases (CVD), mortality associated with CVD comprises more than 30\% of global deaths. ${ }^{1}$

The vast majority of ACS cases result from the rupture of a previously stable coronary plaque unexpectedly becoming vulnerable and unstable. ${ }^{2}$ Prompt identification of vulnerable coronary plaques can lead to timely revascularization of the lesion and plaque sealing with a coronary stent. This protects the plaque from rupture and prevents the development of an acute coronary syndrome. ${ }^{3}$ Such a strategy implies well-timed recognition of a vulnerable plaque, and many studies have been directed at exposing certain plaque features associated with a higher degree of plaque vulnerability.

Plaque vulnerability results from complex pathophysiological mechanisms that lead to changes in plaque structure and morphology. ${ }^{2}$ In unstable coronary plaques the percentage of "unstable components" such as fatty tissue and necrotic core increases, while the amount of "stable components" such as fibro-fatty tissue or calcific tissue decreases. The fibrous cap becomes thinner, predisposing the plaque to rupture.4,5

A vulnerable plaque is defined as a plaque prone to rupture. Because the rupture of the protective fibrous cap exposes the plaque content to circulating blood thrombogenic components, thrombus formation occurs at the site of the lesion. ${ }^{6,7}$

Alterations in plaque structure, indicative of a vulnerable plaque, are identifiable using contemporary imaging technologies including Cardiac Computed Tomography Angiography (CCTA), optical coherence tomography (OCT), intravascular ultrasound (IVUS) and virtual histology intravascular ultrasound (VH-IVUS).

\section{PLAQUE IMAGING — FEATURES OF VULNERABILITY}

A coronary atheromatous plaque becomes unstable on exhibiting a large necrotic lipid core and a thin fibrotic cap, which is invaded by macrophages..$^{8-12}$ There may be associated active vascular remodeling, a process characterized by enlargement of the vessel diameter at the site of the plaque and the presence of spotty calcifications within the plaque. ${ }^{9,13}$

\section{INVASIVE AND NONINVASIVE IMAGING TECHNIQUES FOR PLAQUE CHARACTERIZATION}

CCTA is currently the most common used technology for the noninvasive assessment of coronary atherosclerotic plaques. This technique has the unique ability to identify the presence of low-attenuation atheroma or napkinring sign within the plaque, described as a ring-shaped area with a low density upon CT examination, which is surrounded by a high-density attenuation. These features have been demonstrated to be associated with culprit plaques and a high rate of adverse cardiovascular events. ${ }^{5,9,12,14}$

However, CCTA is not able to detect the thin cap fibroatheroma (TCFA), a relevant feature of plaque instability. ${ }^{8,15}$ For logistical reasons, in the setting of an acute coronary syndrome, it could be difficult to perform complex noninvasive imaging tests such as CСТA, as these unstable patients require urgent revascularization.

Invasive imaging of coronary plaques can be carried out during the revascularization procedure, which allows the detection of several vulnerability markers. IVUS is the invasive technique of choice for the assessment of vascular remodeling and plaque quantification. When associated with virtual histology, the procedure is called VH-IVUS and is considered to be the gold standard for the detection of a necrotic core and for the quantification of plaque components. OCT is the technique of choice for the measurement of fibrous cap thickness, assessment of macrophage content and visualization of intracoronary thrombus. ${ }^{16-20}$

Near-infrared spectroscopy is another invasive imaging method that has been proposed for studying the lipid content of coronary plaques. However, it has not been implemented on a large scale in clinical practice. ${ }^{21,22}$

The aim of this systematic review was to perform a systematic analysis of the studies characterizing vulnerable plaque features visualized using invasive imaging methods such as optical coherence tomography and intravascular ultrasound, in order to identify the most efficient invasive technique that can be used for plaque characterization in patients with acute myocardial infarction.

\section{METHODS}

The present study was performed using the methodology described in the Preferred Reporting Items for Systematic Reviews and Meta-Analyses (PRISMA) guideline together with Cochrane Collaboration and Meta-Analysis of Observational Studies in Epidemiology (MOOSE) recommendations. ${ }^{23,24}$ 


\section{SEARCH STRATEGY AND DATA SOURCES}

The search strategy was directed at the identification of all published data relating to the role of invasive imaging techniques for the characterization of vulnerable plaques. Two independent researchers searched Pubmed/Medline, Thomson Reuters Web of Science and the Cochrane Central databases for articles published in English using the following terms: "optical coherence tomography AND vulnerable plaque", "OCT AND vulnerable plaque", "intravascular ultrasound AND vulnerable plaque" OR "IVUS AND vulnerable plaque". The search included only studies published in the last ten years since OCT and IVUS technologies started to be implemented in the clinical practice only in the last decade.

\section{STUDY SELECTION AND ELIGIBILITY CRITERIA}

This systematic review included all the studies that reported the characteristics of coronary plaques assessed by both intravascular ultrasound and optical coherence tomography, and comparing the results obtained with the two techniques in unstable patients presenting with acute coronary syndromes. Articles that included only OCT or IVUS analysis, or comparison between IVUS and Cardiac CT or between OCT and Cardiac CT, without including both invasive imaging techniques, were excluded from the analysis. Studies addressing only stable coronary plaques or stable patients were also excluded, as were isolated case reports or case series, review articles, editorials, letters, conference meeting abstracts and articles in which a fulltext was not available.

Furthermore, studies analyzing less than thirty coronary plaques, those not providing relevant data, those related to non-coronary vulnerable plaques such as carotid plaques, or only ex-vivo studies were excluded.

\section{DATA EXTRACTION AND ANALYSIS}

Two researchers independently reviewed all the included articles and analyzed the following data: demographic information, baseline characteristics of study populations, and morphologic and anatomic features of unstable plaques, such as: presence of ruptured plaques, presence of thin cap fibroatheroma, macrophage content, extent of lipid-rich plaque volume, stenosis degree, stenosis location, presence and location of culprit coronary plaque.

As none of the studies identified by the literature search provided a complete characterization of all plaque features in different settings associated with the definition of higher vulnerability (vide supra), this systematic review was structured in four parts, according to the plaque characteristics studied by each study group.

Part 1 consisted of the analysis of features associated with vulnerability of coronary plaques with thin cap fibroatheroma versus those with thick cap fibroatheroma, as assessed by OCT and IVUS.

Part 2 consisted of a comparison between IVUS and OCT plaque features in ruptured coronary plaques versus nonruptured coronary plaques.

Part 3 consisted of a comparison between plaque features, as identified by IVUS and OCT, associated with culprit lesions versus those associated with non-culprit lesions.

Part 4 consisted of a comparison between OCT and IVUS-derived features characterizing plaque composition in lipid-rich versus non-lipid-rich plaques, the lipid content being estimated by IVUS attenuation or OCT features.

\section{PLAQUE FEATURES ANALYSED}

The following plaque features were identified:

a) IVUS-derived features:

- external elastic membrane cross-sectional area (EEM CSA) and luminal cross-sectional area (CSA) at the reference site;

- EEM CSA and luminal CSA at the location of the lesion;

- plaque plus media CSA;

- plaque burden;

- remodeling index and the presence of positive remodeling.

b) VH-IVUS-derived features:

- area and volumetric percentage of necrotic core;

- fibrotic tissue;

- fibro-fatty tissue and calcific tissue;

c) OCT-derived features:

- thickness of the fibrous cap;

- lipid arch;

- lipid length;

- presence and extent of microvessels;

- macrophages;

- calcifications;

- plaque rupture;

- TCFA and thrombus.

\section{STATISTICAL ANALYSIS}

The reported variables characterizing coronary plaques are presented as either the mean (SD) for the normally 


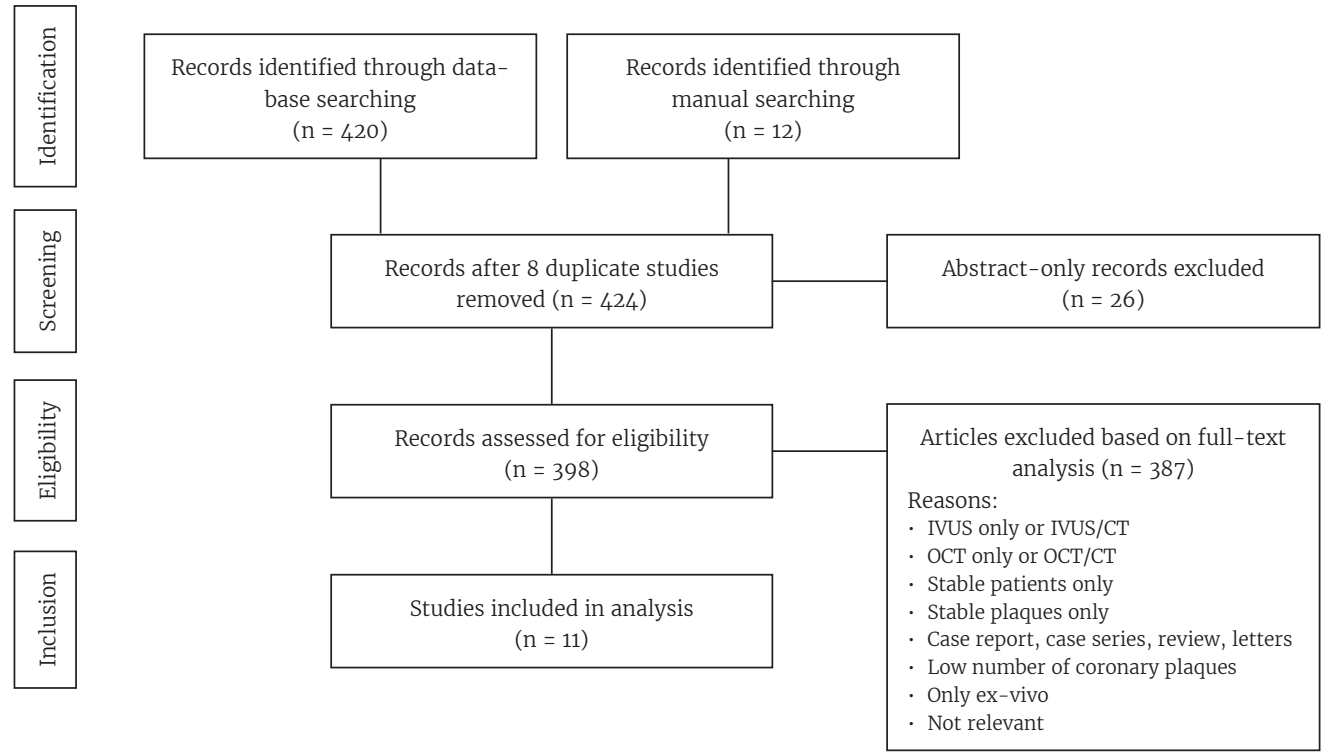

FIGURE 1. Flow chart of the searching process and results of article selection

\section{Characterization of vulnerable coronary plaques assessed by intravascular imaging}

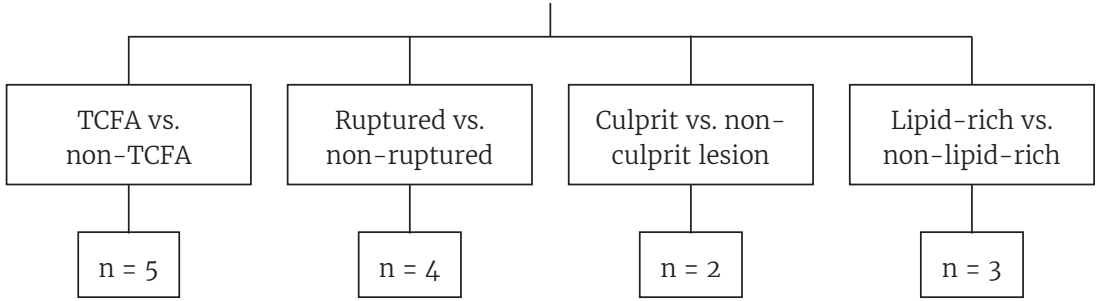

Plaque features

IVUS + VH-IVUS

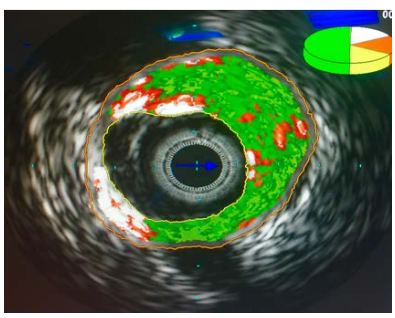

- EEM CSA

- luminal CSA

- plaque plus media CSA

- plaque burden

- remodeling index

- positive remodeling

- necrotic core

- fibrotic tissue

- fibro-fatty tissue

- calcific tissue
OCT

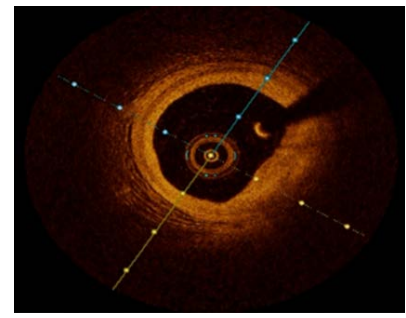

- fibrous cap thickness

- lipid arch

- lipid length

- microvessels

- machrophages

- calcifications

- plaques rupture

- TCFA

- thrombus

FIGURE 2. Design of the systematic review on assessment of vulnerable plaque features using intracoronary imaging 
TABLE 1. Characteristics of studies that assessed plaque vulnerability features using intracoronary imaging

\begin{tabular}{|c|c|c|c|c|}
\hline Study author & $\begin{array}{c}\text { Number of patients } \\
\text { included }\end{array}$ & $\begin{array}{c}\text { Number of plaques } \\
\text { analyzed }\end{array}$ & Age (mean \pm SD) & Clinical setting \\
\hline Sawada et al. ${ }^{15}$ & 56 & 126 & $70.1 \pm 8.2$ vs. $68.1 \pm 8.7$ & TCFA vs. non-TCFA \\
\hline Tian et al. ${ }^{20}$ & 82 & 266 & $55.6 \pm 9.4$ & ruptured culprit, ruptured non-culprit, TCFA \\
\hline Roleder et al. ${ }^{22}$ & 60 & 76 & $59 \pm 10$ vs. $62 \pm 11$ & TCFA vs. non-TCFA \\
\hline Kato et al. ${ }^{27}$ & 75 & 75 & $66.5 \pm 10.0$ vs. $69.4 \pm 9.7$ & ruptured vs. non-ruptured plaque \\
\hline Kwon et al. ${ }^{28}$ & 133 & 133 & $60.11 \pm 12.5$ vs. $61.9 \pm 9.9$ & ruptured vs. non-ruptured \\
\hline Maejima et al. ${ }^{29}$ & 150 & 150 & $67.9 \pm 10.9$ vs. $66.0 \pm 10.7$ & culprit vs. non-culprit \\
\hline Kubo et al. ${ }^{30}$ & 104 & 104 & $75 \pm 7$ vs. $73 \pm 7$ & $\begin{array}{l}\text { LR vs. non-LR plaque } \\
\text { (attenuated vs. non-attenuated plaque) }\end{array}$ \\
\hline
\end{tabular}

TCFA - thin cap fibro-atheroma, LR - lipid-rich

distributed variables or as the mean and median $\left(25^{\text {th }}\right.$ to $75^{\text {th }}$ percentiles) for the non-normally distributed variables, as reported in the original sources.

Categorical variables are presented as absolute frequencies and percentages.

\section{RESULTS}

\section{RESULTS OF THE DATABASE SEARCH}

A total number of 432 studies were identified, out of which 420 through database searching and 12 through manual searching. After eight duplicate studies had been removed, 424 studies were screened. Twenty-six studies were excluded, as they were available in abstract-only form, re- sulting in 398 studies being checked for eligibility. Only eleven studies fulfilled the eligibility criteria and were included in the analysis (Figure 1).

A total number of 1,568 coronary plaques from 1,225 patients with acute coronary syndromes who underwent both IVUS and OCT for analysis of plaque features, were analyzed (Table 1). The structure of the systematic review is summarized in Figure 2.

Part 1 of the systematic review addressed the topic of TCFA in vulnerable coronary plaques. It consisted of the analysis of five studies reporting on a total of 368 coronary plaques assessed by OCT and IVUS in $261 \mathrm{pa-}$ tients. ${ }^{15,19,20,22,25}$

Part 2 compared the IVUS- and OCT-derived plaque characteristics in 587 ruptured versus non-ruptured cor-

TABLE 2. Baseline study and overall population characteristics of the patients included in the 11 studies and in the four parts of the systematic review

\begin{tabular}{|c|c|c|c|c|c|}
\hline & Total & $\begin{array}{c}\text { Part } 1 \\
\text { TCFA vs. non-TCFA }\end{array}$ & $\begin{array}{c}\text { Part } 2 \\
\text { ruptured vs. non- } \\
\text { ruptured } \mathrm{CP}\end{array}$ & $\begin{array}{c}\text { Part } 3 \\
\text { culprit vs. non-cul- } \\
\text { prit CP }\end{array}$ & $\begin{array}{c}\text { Part } 4 \\
\text { lipid-rich vs. non- } \\
\text { lipid-rich CP }\end{array}$ \\
\hline n studies & 11 & 5 & 4 & 2 & 3 \\
\hline n patients & 1101 & 261 & 608 & 232 & 302 \\
\hline $\mathrm{n}$ plaques studied & 1303 & 368 & 587 & 173 & 302 \\
\hline Male, n (\%) & $911(76)$ & $181(69)$ & $478(79)$ & $171(73)$ & $228(75)$ \\
\hline Smokers, n (\%) & $480(40)$ & $94(36)$ & $273(45)$ & $96(41)$ & $121(40)$ \\
\hline Diabetes mellitus, n (\%) & $411(35)$ & $121(46)$ & $198(33)$ & $96(41)$ & $104(34)$ \\
\hline Hypertension, n (\%) & $787(66)$ & $187(72)$ & $366(60)$ & $139(60)$ & $222(73)$ \\
\hline Hyperlipidemia, n (\%) & $599(50)$ & $177(68)$ & $250(41)$ & $139(60)$ & $160(53)$ \\
\hline
\end{tabular}




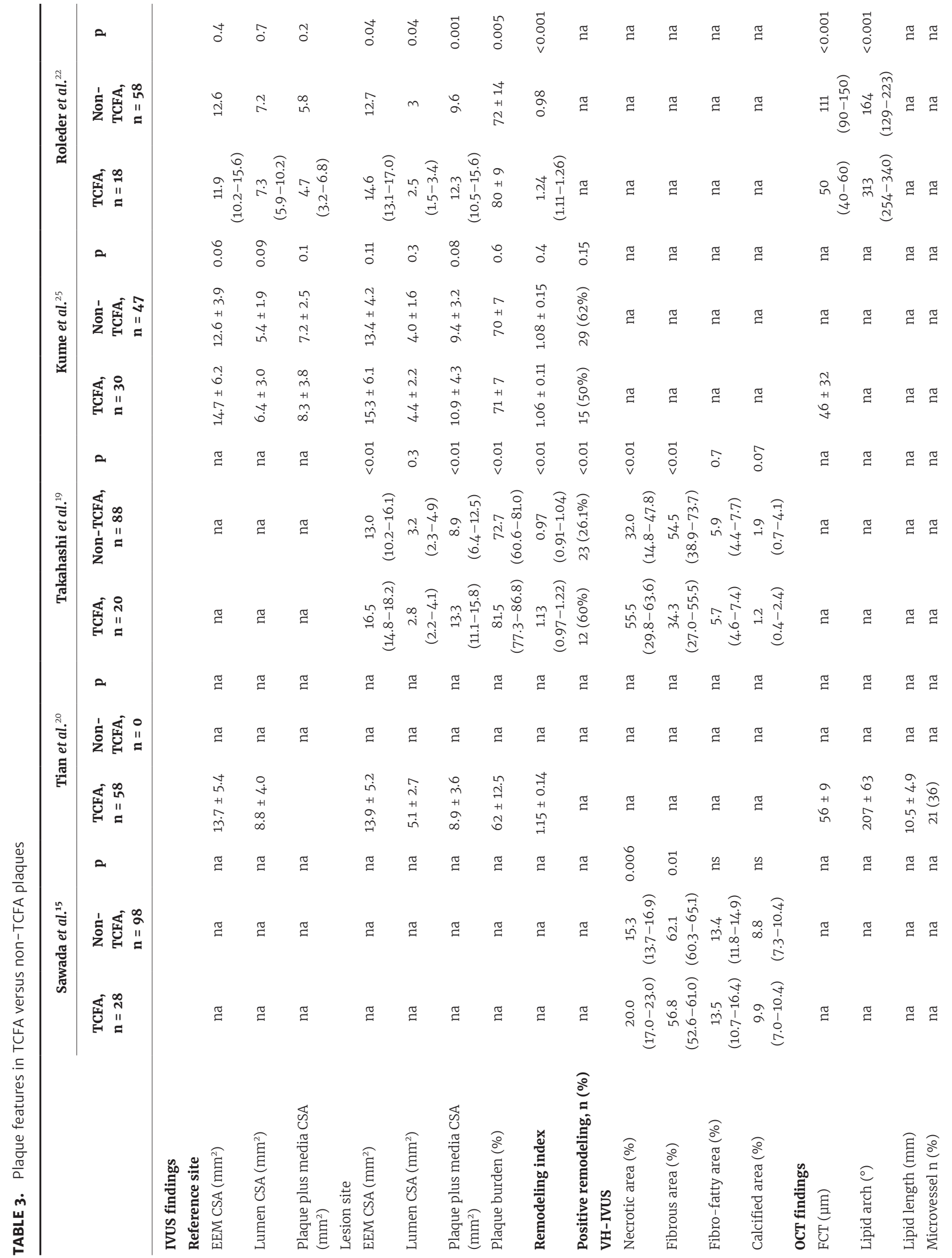




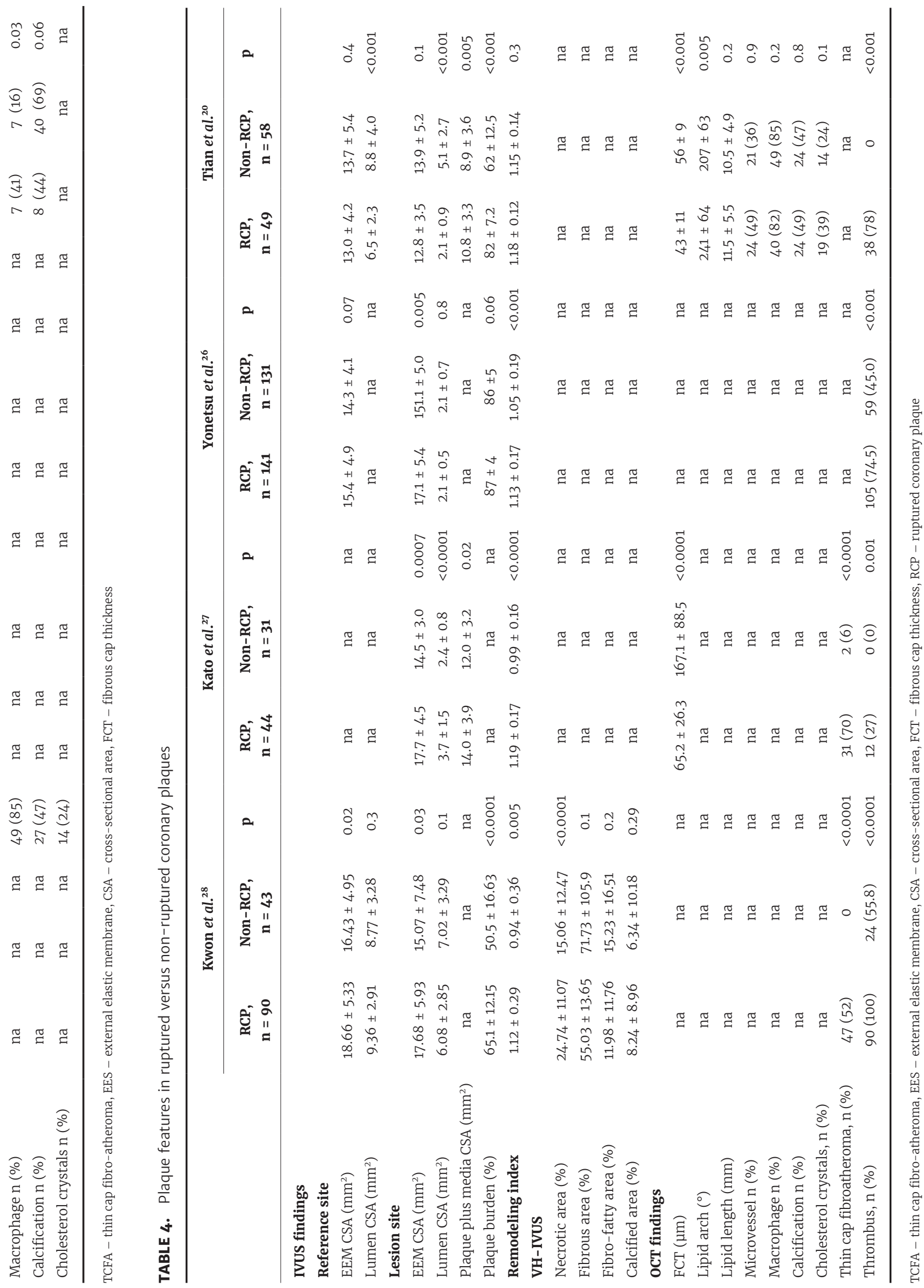


TABLE 5. Plaque features in culprit versus non-culprit lesions

\begin{tabular}{|c|c|c|c|c|c|c|}
\hline & \multicolumn{3}{|c|}{ Maejima et al. ${ }^{29}$} & \multicolumn{3}{|c|}{ Tian et al..$^{20}$} \\
\hline & $\begin{array}{c}\mathrm{CP} \\
\mathrm{n}=\mathbf{7 3}\end{array}$ & $\begin{array}{c}\text { Non-CP, } \\
n=32\end{array}$ & $\mathbf{p}$ & $\begin{array}{c}\mathrm{CP} \\
\mathrm{n}=44\end{array}$ & $\begin{array}{c}\text { Non-CP } \\
n=19\end{array}$ & $\mathbf{p}$ \\
\hline \multicolumn{7}{|l|}{ IVUS findings } \\
\hline \multicolumn{7}{|l|}{ Reference site } \\
\hline EEM CSA $\left(\mathrm{mm}^{2}\right)$ & na & na & na & $13.0 \pm 4.2$ & $12.3 \pm 4.4$ & 0.5 \\
\hline Lumen CSA $\left(\mathrm{mm}^{2}\right)$ & na & na & na & $6.5 \pm 2.3$ & $7.2 \pm 3.3$ & 0.4 \\
\hline Lesion site & na & na & na & & & \\
\hline $\operatorname{EEM~CSA~}\left(\mathrm{mm}^{2}\right)$ & $14.4 \pm 4.6$ & $12.3 \pm 4.3$ & 0.01 & $12.8 \pm 3.5$ & $12.6 \pm 4.6$ & 0.8 \\
\hline Lumen CSA $\left(\mathrm{mm}^{2}\right)$ & $1.6 \pm 0.4$ & $2.0 \pm 0.9$ & 0.06 & $2.1 \pm 0.9$ & $4.6 \pm 2.3$ & 0.001 \\
\hline Plaque plus media CSA $\left(\mathrm{mm}^{2}\right)$ & $12.8 \pm 4.6$ & $10.3 \pm 3.9$ & 0.003 & $10.8 \pm 3.3$ & $8.0 \pm 2.8$ & 0.001 \\
\hline Plaque burden (\%) & $87 \pm 5$ & $82 \pm 8$ & $<0.001$ & $82 \pm 7.2$ & $64 \pm 7.2$ & $<0.001$ \\
\hline Remodeling index & $10.5 \pm 0.16$ & $0.92 \pm 0.16$ & 0.002 & $1.18 \pm 0.12$ & $1.18 \pm 0.13$ & 0.8 \\
\hline \multicolumn{7}{|l|}{ OCT findings } \\
\hline $\mathrm{FCT}(\mu \mathrm{m})$ & 60 & 82 & $<0.001$ & $43 \pm 11$ & $41 \pm 10$ & 0.2 \\
\hline Lipid $\operatorname{arch}\left({ }^{\circ}\right)$ & na & na & na & $241 \pm 64$ & $214 \pm 54$ & 0.02 \\
\hline Lipid length (mm) & na & na & na & $11.5 \pm 5.5$ & $10.5 \pm 2.8$ & 0.4 \\
\hline Microvessel n (\%) & na & na & na & $24(49)$ & $7(37)$ & 0.1 \\
\hline Macrophage n (\%) & na & na & na & $40(82)$ & $14(74)$ & 0.4 \\
\hline Calcification n (\%) & na & na & na & $24(49)$ & $6(32)$ & 0.09 \\
\hline Cholesterol crystals, n (\%) & $38(52)$ & $8(25)$ & $<0.01$ & na & na & na \\
\hline Thin cap fibroatheroma, n (\%) & $49(67)$ & $13(41)$ & $<0.01$ & na & na & na \\
\hline Thrombus, n (\%) & $63(86)$ & $5(16)$ & $<0.01$ & $38(78)$ & $12(63)$ & 0.2 \\
\hline
\end{tabular}

TCFA - thin cap fibro-atheroma, EES - external elastic membrane, CSA - cross-sectional area, FCT - fibrous cap thickness, CP - culprit plaque

onary plaques, described in four studies on 608 patients with acute coronary syndromes. ${ }^{20,26,27,28}$

Part 3 consisted of the analysis of two studies reporting on OCT- and IVUS-derived plaque characteristics of 173 culprit and non-culprit coronary plaques from $232 \mathrm{pa}-$ tients with acute coronary syndromes. ${ }^{20,29}$

Part 4 consisted of the analysis of data on the lipid content of 302 plaques from 302 patients. ${ }^{19,30,31}$

\section{CHARACTERISTICS OF THE PATIENTS AND THE STUDIES}

Baseline study and overall population characteristics of the patients included in the eleven studies and the four parts of this review are presented in Table 2.

\section{PLAQUE FEATURES IN TCFA VERSUS NON-TCFA PLAQUES}

IVUS studies comparing plaque features in TCFA versus non-TCFA plaques showed that plaque burden $(\mathrm{p}<0.01)$ and the remodeling index $(\mathrm{p}<0.01)$ were significantly higher in plaques with TCFA compared with those with non-TCFA. At the same time, the percentage of necrotic core was significantly greater in TCFA plaques $(\mathrm{p}<0.01)$, indicating that all these features are valuable imagingbased biomarkers for predicting plaque vulnerability. Interestingly, the cross-sectional area of the external elastic membrane at the site of the lesion was significantly higher in patients with more vulnerable plaques, having a TCFA. Only one study analysed OCT features in TCFA vs. non-TCFA plaques, indicating a significantly lower thickness of the fibrous cap $(\mathrm{p}<0.0001)$, a significantly larger lipid arch $(\mathrm{p}<0.001)$ and a significantly higher percent of macrophage content $(\mathrm{p}=0.03)$ in plaques with TCFA compared with plaques with non-TCFA (Table 3 ).

\section{PLAQUE FEATURES IN RUPTURED VERSUS NON-RUPTURED CORONARY PLAQUES}

Four studies analysed IVUS and OCT plaque features in ruptured versus non-ruptured coronary plaques (Table 4). Similarly with the results obtained when analysing TCFA plaques, all these studies identified plaque burden ( $p<0.001)$ and remodeling index $(p=0.005)$ as being significantly increased in patients with ruptured plaques. VH-IVUS identified the percentage of necrotic core as be- 
TABLE 6. Plaque features in lipid-rich versus non-lipid-rich plaques

\begin{tabular}{|c|c|c|c|c|c|c|c|c|c|}
\hline & \multicolumn{3}{|c|}{ Lee et $a l .{ }^{31}$} & \multicolumn{3}{|c|}{ Kubo et al. ${ }^{30}$} & \multicolumn{3}{|c|}{ Takahashi et al. ${ }^{19}$} \\
\hline & $\begin{array}{c}\text { LR, } \\
\mathrm{n}=47\end{array}$ & $\begin{array}{c}\text { Non-LR, } \\
\mathrm{n}=\mathbf{8 8}\end{array}$ & $\mathbf{p}$ & $\begin{array}{c}\mathrm{LR} \\
\mathrm{n}=\mathbf{4 1}\end{array}$ & $\begin{array}{c}\text { Non-LR, } \\
n=63\end{array}$ & $\mathbf{p}$ & $\begin{array}{c}\text { LR, } \\
\mathrm{n}=56\end{array}$ & $\begin{array}{c}\text { Non-LR, } \\
n=52\end{array}$ & $\mathbf{p}$ \\
\hline \multicolumn{10}{|l|}{ IVUS findings } \\
\hline \multicolumn{10}{|l|}{ Reference site } \\
\hline $\operatorname{EEM}$ CSA $\left(\mathrm{mm}^{2}\right)$ & $17.9 \pm 5.1$ & $14.1 \pm 4.3$ & $<0.001$ & $12.5 \pm 5.3$ & $10.9 \pm 4.4$ & 0.1 & na & na & na \\
\hline Lumen CSA $\left(\mathrm{mm}^{2}\right)$ & na & na & na & $8.6 \pm 3.7$ & $7.4 \pm 3.3$ & 0.1 & na & na & na \\
\hline \multicolumn{10}{|l|}{ Lesion site } \\
\hline $\operatorname{EEM}$ CSA $\left(\mathrm{mm}^{2}\right)$ & $18.9 \pm 5.9$ & $14.2 \pm 5.3$ & $<0.001$ & $12.4 \pm 5.0$ & $10.1 \pm 4.4$ & 0.01 & $15.7(13.1-19.3)$ & $\begin{array}{c}11.4(9.8- \\
14.1)\end{array}$ & $<0.01$ \\
\hline Lumen CSA $\left(\mathrm{mm}^{2}\right)$ & $2.24 \pm 0.65$ & $2.25 \pm 5.2$ & $<0.001$ & $2.5 \pm 1.0$ & $2.6 \pm 1.0$ & 0.6 & $2.9(2.2-4.2)$ & $3.5(2.4-4.9)$ & 0.3 \\
\hline Plaque plus media CSA $\left(\mathrm{mm}^{2}\right)$ & na & na & na & $9.9 \pm 4.6$ & $7.5 \pm 4.0$ & 0.006 & $12.5(9.6-15.1)$ & $8.0(5.8-9.5)$ & $<0.01$ \\
\hline Plaque burden (\%) & $87.4 \pm 4.1$ & $82.7 \pm 6.4$ & $<0.0001$ & $78 \pm 10$ & $73 \pm 9$ & 0.02 & $80.4(68.8-83.9)$ & $69.1(57.9-78.8)$ & $<0.01$ \\
\hline Remodeling index & $1.06 \pm 0.19$ & $1.01 \pm 0.18$ & 0.09 & na & na & na & $1.02(0.91-1.15)$ & $0.97(0.92-1.01)$ & $<0.01$ \\
\hline Positive remodeling, $\mathbf{n}(\%)$ & $25(55.3)$ & $34(38.6)$ & 0.09 & $22(54)$ & $19(30)$ & 0.01 & $28(50.0)$ & $7(13.5)$ & $<0.01$ \\
\hline \multicolumn{10}{|l|}{ VH-IVUS } \\
\hline Necrotic core volume (\%) & na & na & na & na & na & na & $33.0(22.3-42.8)$ & $19.0(11.0-33.0)$ & $<0.01$ \\
\hline Fibrous volume (\%) & na & na & na & na & na & na & $51.5(45.5-63.8)$ & $67.5(53.8-77.3)$ & $<0.01$ \\
\hline Fibro-fatty volume (\%) & na & na & na & na & na & na & $6.0(5.0-7.0)$ & $5.0(4.0-6.0)$ & $<0.01$ \\
\hline Calcified volume (\%) & na & na & na & na & na & na & $1.0(1.0-2.0)$ & $2.0(1.0-3.0)$ & 0.05 \\
\hline \multicolumn{10}{|l|}{ OCT findings } \\
\hline $\mathrm{FCT}(\mu \mathrm{m})$ & $60(50-100)$ & $85(60-120)$ & 0.005 & $103 \pm 70$ & $145 \pm 97$ & 0.04 & na & na & na \\
\hline Ruptured plaque, n (\%) & $16(34.0)$ & $16(18.2)$ & 0.06 & $18(44)$ & $7(11)$ & $<0.001$ & na & na & na \\
\hline Thin cap fibroatheroma, n (\%) & $24(51.1)$ & $19(21.6)$ & $<0.0001$ & $20(48)$ & $10(16)$ & $<0.001$ & na & na & na \\
\hline Thrombus, n (\%) & $6(12.8)$ & $16(18.2)$ & 0.4 & $22(54)$ & $11(17)$ & $<0.001$ & $105(74.5)$ & $59(45.0)$ & $<0.001$ \\
\hline
\end{tabular}

TCFA - thin cap fibro-atheroma, EES - external elastic membrane, CSA - cross-sectional area, FCT - fibrous cap thickness, LR - lipid-rich

ing significantly increased in ruptured coronary plaques as compared to non-ruptured ones ( $p<0.0001)$. EEM CSA at the lesion site was significantly larger in patients with ruptured plaques in three of the IVUS studies $(\mathrm{p}=0.03, \mathrm{p}$ $=0.0007$ and $\mathrm{p}=0.005$, respectively), while there were no differences between EEM CSA at the reference site. OCT analysis revealed a significantly thinner fibrous cap in the ruptured plaques $(\mathrm{p}<0.001)$, with a higher incidence of TCFA ( $\mathrm{p}<0.0001)$ in these extremely unstable lesions, accompanied by the presence of thrombus in a significantly greater extent in all the studies ( $\mathrm{p}<0.001)$.

\section{PLAQUE FEATURES IN CULPRIT VERSUS NON-CULPRIT LESIONS}

In the two studies included in this analysis, IVUS and OCT examinations identified the following features as significantly associated with culprit lesions (Table 5):

- a higher EEM CSA at lesion level ( $p=0.01)$;

- a lower luminal CSA ( $\mathrm{p}=0.001)$;

- greater plaque burden ( $\mathrm{p}<0.0001)$ shown by IVUS;

- the presence of TCFA $(\mathrm{p}<0.01)$;
- $\operatorname{lipid} \operatorname{arch}(\mathrm{p}=0.02)$;

- plaque rupture $(\mathrm{p}<0.01)$;

- thrombus $(\mathrm{p}<0.01)$ shown by OCT.

\section{PLAQUE FEATURES IN LIPID-RICH VERSUS NON-LIPID-RICH PLAQUES}

In all three studies including lipid-rich vs. non-lipidrich plaques, a higher EEM CSA at the lesion level ( $p$ $<0.001, p=0.01$ and $p<0.001$, respectively) and a higher plaque burden ( $\mathrm{p}<0.0001, \mathrm{p}=0.02$ and $\mathrm{p}<0.01$, respectively) as shown by IVUS, were associated with lipid-rich plaques, as well as a thinner fibrous cap $(\mathrm{p}=0.005$ and $\mathrm{p}$ $=0.04$, respectively), presence of TCFA $(p<0.001)$, ruptured plaques $(\mathrm{p}<0.001)$ or thrombus by OCT $(\mathrm{p}<0.001)$ (Table 6). In two studies the remodeling index was significantly higher in lipid-rich plaques ( $\mathrm{p}<0.001)$ and one study, which assessed plaque composition by VH-IVUS, identified significant differences between LR plaques and non-LR ones ( $p<0.001$ for necrotic core, $<0.01$ for fibrotic tissue, $<0.001$ for fibro-fatty tissue and 0.05 for calcified tissue). 


\section{DISCUSSION}

Vulnerable coronary plaques are characterized by modifications of plaque components leading to a change in their morphology. As a consequence of these changes, vulnerable coronary plaques display specific signatures of vulnerability that are easily identified by imaging techniques such as CCTA, OCT or IVUS. $8,9,32,33$ These signature features have been demonstrated to be associated with culprit plaques in ACS and with higher rates of adverse events in follow-up studies. ${ }^{33,34}$

Since the introduction of modern CCTA technologies, there has been a tendency to replace invasive imaging for assessment of plaque characteristics with noninvasive assessment techniques. CCTA is also able to provide relevant information on the calcium content of the entire coronary tree and also of each coronary artery, a parameter with a high prognostic value. ${ }^{8}$

However, a significant proportion of vulnerable coronary plaques are identified in patients with ACS, a group in which the delay associated with performing CCTA should be avoided. It has been demonstrated that in acute myocardial infarction, the augmented inflammation accompanying the infarction leads to a generalized pan-coronary vulnerability that is associated with the development of new coronary events originating from the site of nonculprit plaques. ${ }^{2,34}$

An evaluation of the total coronary plaque burden and associated lesions is necessary, as ACS patients may present secondary rupture of non-culprit plaques. In a study that aimed to investigate the IVUS-derived biomarkers and coronary outcomes, it was suggested that an increased plaque burden is a predictor for future major adverse cardiovascular events..$^{35} \mathrm{~A}$ substudy of the PROSPECT trial sought to analyze the frequency, patient- and lesionrelated features of non-culprit plaques that ruptured, in patients with ACS undergoing emergency PCI. The results showed that patients with ACS and non-culprit plaque rupture had a higher atherosclerotic burden compared to those with non-culprit lesions with no rupture $(66.0 \%$ [95\% CI: $64.5-67.4 \%$ ] vs. $56.0 \%$ [ $95 \%$ CI: $55.6-56.4 \%$ ]; p $<0.0001)$. Nevertheless, patients with non-culprit plaque rupture presented a higher rate of fibro-atheromatous plaques with a residual necrotic core, but under optimal medical therapy their overall rate of cardiovascular adverse outcome was not higher during a mean follow-up of 21 months. ${ }^{34}$

Therefore, the identification of vulnerability features at the site of coronary plaques from the entire coronary tree during the revascularization procedure, could help to prevent the development of new episodes of ACS. This assessment of coronary plaques in an ACS patient should be performed using on-site invasive imaging technology, concomitant with the revascularization procedure.

\section{PLAQUE FEATURES ASSOCIATED WITH VULNERABILITY}

Currently, there are several studies regarding the noninvasive assessment of plaque morphology and composition, which have identified specific imaging biomarkers that are suggestive for plaque vulnerabilization. In a study on CT imaging characteristics of high-risk coronary plaques, it was reported that a low-density plaque with spotty calcifications associated with positive vessel remodeling is linked to a higher risk of developing an acute coronary syndrome. ${ }^{9,13}$

In the current analysis, two studies identified a new parameter, the attenuated plaque by IVUS, as a feature of plaque vulnerability. ${ }^{19,31}$ In the scope of this systematic review, these attenuated plaques were considered as lipidrich plaques, as a higher fatty content is associated with lower plaque density at both CCTA and IVUS.,12

While a large number of studies used IVUS-alone or OCT-alone assessment of coronary plaques, or compared these two techniques with CCTA, few studies addressed vulnerable plaque characterization using both technologies. In an emergency setting, it may be difficult for an operator to choose between the two techniques, as to a significant extent, the information revealed by the two techniques are complementary, and the utilization of both techniques would lead to a significant increase in procedure cost. While IVUS examination can provide a useful quantification of plaque size and components, OCT is gold-standard for the measurement of the fibrous cap, one of the most significant predictors of plaque vulnerability, and is the only method that can visualize, with a good resolution, macrophage infiltration, microvessels and intracoronary thrombi. In a comparative study of IVUS and OCT in patients with ACS, it was reported that intracoronary thrombi, resulting from the rupture of a culprit plaque, could be identified more often using OCT than with IVUS. ${ }^{27}$ In contrast, OCT is not considered suitable for identifying vascular remodeling of the culprit plaques, even though it can assess the coronary wall with a higher degree of accuracy than IVUS.

This systematic review identified that IVUS-derived features significantly correlated with plaque vulnerability were plaque burden, remodeling index, EEM CSA and the amount of necrotic core, while OCT-derived features characterizing unstable plaque were TCFA, lipid arch, ac- 
cumulation of macrophages and the presence of intracoronary thrombus.

However, the consensus on IVUS-derived plaque features was higher than the one recorded in OCT-derived plaque features, mainly because all IVUS analyses included the same plaque features, while OCT analyses addressed different ones, making it relatively hard to generalize their conclusion.

\section{CONCLUSION}

Both IVUS and OCT are invasive imaging techniques able to provide relevant information on the vulnerability of coronary atheromatous plaque by identifying various plaque features that have been proved to be significantly associated with unstable plaques. Information from the two techniques are complementary, and both can serve as a useful clinical diagnostic measure, especially in cases of ACS patients undergoing a revascularization procedure.

\section{ACKNOWLEDGEMENT}

This research was supported via the research grant no. 103544/2016, contract number 26/01.09.2016, financed by the Romanian Ministry of European Funds, the Romanian Government and the European Union.

\section{CONFLICT OF INTEREST}

Nothing to declare.

\section{REFERENCES}

1. World Health Organisation, Cardiovascular disease (CVDs), 2016. Available from: http://www.who.int/mediacentre/ factsheets/fs317/en/\#.

2. Choy SY, Mintz GS. What have we learned about plaque rupture in acute coronary syndromes? CurrCardiol Rep. 2010;12:338343. doi: 10.1007/s11886-010-0113-x.

3. Giblett JP, Brown AJ, Keevil H, Jaworski C, Hoole SP, West NE. Implantation of bioresorbable vascular scaffolds following acute coronary syndrome is associated with reduced early neointimal growth and strut coverage. EuroIntervention. 2016;12:724-733. doi: 10.4244/EIJV12I6A117.

4. Pundziute G, Schuijf JD, Jukema JW, et al. Evaluation of plaque characteristics in acute coronary syndromes: noninvasive assessment with multi-slice computed tomography and invasive evaluation with intravascular ultrasound radiofrequency data analysis. Eur Heart J. 2008;29:2373-2381. doi: 10.1093/eurheartj/ehn356.

5. Finn AV, Nakano M, Narula J, Kolodgie FD, Virmani R. Concept of vulnerable/unstable plaque. Arterioscler Thromb Vasc Biol. 2010;30:1282-1292. doi: 10.1161/ATVBAHA.108.179739.
6. Andreou I, Antoniadis AP, Shishido K, et al. How do we prevent the vulnerable atherosclerotic plaque from rupturing? Insights from in vivo assessments of plaque, vascular remodeling, and local endothelial shear stress. J Cardiovasc Pharmacol Ther. 2015;20:261-275. doi: 10.1177/1074248414555005.

7. Bentzon JF, Otsuka F, Virmani R, Falk E. Mechanisms of plaque formation and rupture. Circ Res. 2014;114:1852-1866. doi: 10.1161/CIRCRESAHA.114.302721.

8. Owen DRJ, Lindsay AC, Choudhury RP, Fayad ZA. Imaging of Atherosclerosis. Annual review of medicine. 2011;62:25-40. doi:10.1146/annurev-med-041709-133809.

9. Motoyama S, Masayoshi S, Harigaya H, et al. Computed tomographic angiography characteristics of atherosclerotic plaques subsequently resulting in acute coronary syndrome. J Am Coll Cardiol. 2009;54:49-57. doi: 10.1016/j. jacc.2009.02.068.

10. Sinclair H, Bourantas C, Bagnall A, Mintz GS, Kunadian V. OCT for identification of Vulnerable Plaque in Acute Coronary Syndrome. JACC Cardiovasc Imaging. 2015;8:198-209. doi: 10.1016/j.jcmg.2014.12.005.

11. Batty JA, Subba S, Luke P, Gigi LWC, Sinclair H, Kunadian V. Intracoronary imaging in the detection of vulnerable plaques. Curr Cardiol Rep. 2016;18:28. doi: 10.1007/s11886016-0705-1.

12. Dalager MG, Bøttcher M, Thygesen J, Andersen G, Bøtker HE. Different Plaque Composition and Progression in Patients with Stable and Unstable Coronary Syndromes Evaluated by Cardiac CT. BioMed Research International. 2015;2015:401357. doi:10.1155/2015/401357.

13. Thomsen C, Abdulla J. Characteristics of high-risk coronary plaques identified by computed tomographic angiography and associated prognosis: a systematic review and meta-analysis. Eur Heart J Cardiovasc Imaging. 2016;17:120-129. doi: 10.1093/ ehjci/jev325.

14. Maurovich-Horvat P, Schlett CL, Alkadhi $\mathrm{H}$, et al. The napkin-ring sign indicates advanced atherosclerotic lesions in coronary CT angiography. JACC Cardiovasc Imaging. 2012;5:1243-1252. doi: 10.1016/j.jcmg.2012.03.019.

15. Sawada T, Shite J, Garcia-Garcia HM, et al. Feasibility of combined use of intravascular ultrasound radiofrequency data analysis and optical coherence tomography for detecting thin-cap fibroatheroma. Eur Heart J. 2008;29:1136-1146. doi: 10.1093/eurheartj/ehn132.

16. Jang IK. Optical Coherence Tomography or Intravascular Ultrasound? JACC: Cardiovascular Interventions. 2011;4:492494. doi: 10.1016/j.jcin.2011.02.004.

17. Kajander OA, Pinilla-Echeverri N, Jolly SS, et al. Culprit plaque morphology in STEMI - an optical coherence tomography study: insights from the TOTAL-OCT substudy. EuroIntervention. 2016;12:716-723. doi: 10.4244/EIJV12I6A116.

18. Finn AV, Chandrashekhar Y, Narula J. Vulnerable plaques: from PROSPECT to prospects... JACC Cardiovasc Imaging. 2012;5:334-336. doi: 10.1016/j.jcmg.2012.02.004.

19. Takahashi K, Kakuta T, Yonetsu T, et al. In vivo detection of lipid-rich plaque by using a 40-MHz intravascular ultrasound: a comparison with optical coherence tomography findings. Cardiovasc Interv Ther. 2013;28:333-343. doi: 10.1007/s12928013-0177-9.

20. Tian J, Ren X, Vergallo R, et al. Distinct morphological features of ruptured culprit plaque for acute coronary events compared 
to those with silent rupture and thin-cap fibroatheroma: a combined optical coherence tomography and intravascular ultrasound study. J Am Coll Cardiol. 2014;63:2209-2216. doi: 10.1016/j.jacc.2014.01.061.

21. Caplan JD, Waxman S, Nesto RW, Muller JE. Near-infrared spectroscopy for the detection of vulnerable coronary artery plaques. J Am Coll Cardiol. 2006;47(8 Suppl):C92-C96. doi: 10.1016/j.jacc.2005.12.045.

22. Roleder T, Kovacic JC, Ali Z, Sharma R, et al. Combined NIRS and IVUS imaging detects vulnerable plaque using a single catheter system: a head-to-head comparison with OCT. EuroIntervention. 2014;10:303-311. doi: 10.4244/EIJV10I3A53.

23. Moher D, Liberati A, Tetzlaff J, Altman DG, PRISMA Group. Preferred reporting items for systematic reviews and metaanalyses: the PRISMA statement. BMJ. 2009;339:b2535. doi: 10.1136/bmj.b2535

24. Stroup DF, Berlin JA, Morton SC, et al. Meta-analysis of observational studies in epidemiology: a proposal for reporting. Meta-analysis Of Observational Studies in Epidemiology (MOOSE) group. JAMA. 2000;283:2008-2012.

25. Kume T, Okura H, Yamada R, et al. Frequency and spatial distribution of thin-cap fibroatheroma assessed by 3-vessel intravascular ultrasound and optical coherence tomography: an ex vivo validation and an initial in vivo feasibility study. Circ J. 2009;73:1086-1091.

26. Yonetsu T, Lee T, Murai T, et al. Plaque morphologies and the clinical prognosis of acute coronary syndrome caused by lesions with intact fibrous cap diagnosed by optical coherence tomography. Int J Cardiol. 2016;203:766-774. doi: 10.1016/j. ijcard.2015.11.030

27. Kato M, Dote K, Sasaki S, et al. Presentations of acute coronary syndrome related to coronary lesion morphologies as assessed by intravascular ultrasound and optical coherence tomography. Int J Cardiol. 2013;165:506-511. doi: 10.1016/j. ijcard.2011.09.032.
28. Kwon JE, Lee WS, Mintz GS, et al. Multimodality Intravascular Imaging Assessment of Plaque Erosion versus Plaque Rupture in Patients with Acute Coronary Syndrome. Korean Circulation Journal. 2016;46:499-506. doi: 10.4070/kcj.2016.46.4.499.

29. Maejima N, Hibi K, Saka K, et al. Morphological features of non-culprit plaques on optical coherence tomography and integrated backscatter intravascular ultrasound in patients with acute coronary syndromes. Eur Heart J Cardiovasc Imaging. 2015;16:190-197. doi: 10.1093/ehjci/jeu173.

30. Kubo T, Matsuo Y, Ino Y, et al. Optical Coherence Tomography Analysis of Attenuated Plaques Detected by Intravascular Ultrasound in Patients with Acute Coronary Syndromes. Cardiology Research and Practice. 2011; doi. org/10.4061/2011/687515.

31. Lee T, Kakuta T, Yonetsu T, et al. Assessment of EchoAttenuated Plaque by Optical Coherence Tomography and Its Impact on Post-Procedural Creatine Kinase-Myocardial Band Elevation in Elective Stent Implantation. JACC Cardiovasc Interv. 2011;4:483-491. doi: 10.1016/j.jcin.2010.12.013.

32. Jonathan A. Batty, Subba S, Luke P, Li Wing Chi G, Sinclair $\mathrm{H}$, and Kunadian V. Intravascular detection of the vulnerable plaque. Circ Cardiovasc Imaging. 2011;4:169-78. doi: 10.1007/ s11886-016-0705-1.

33. Suh WM, Seto AH, Margey RJ, Cruz-Gonzalez I, Jang IK. Intravascular detection of the vulnerable plaque. Circ Cardiovasc Imaging. 2011;4:169-178. doi: 10.1161/ CIRCIMAGING.110.958777.

34. Xie Y, Mintz G, Yang J, et al. Clinical Outcome of Nonculprit Plaque Ruptures in Patients with Acute Coronary Syndrome in the PROSPECT Study. 2014. JACC Cardiovasc Imaging. 2014;7:397-405. doi: 10.1016/j.jcmg.2013.10.010.

35. Nicholls SJ, Hsu A, Wolski K, et al. Intravascular ultrasoundderived measures of coronary atherosclerotic plaque burden and clinical outcome. J Am Coll Cardiol. 2010;55:2399-2407. doi: 10.1016/j.jacc.2010.02.026. 